\title{
Spontaneous Spinal Subarachnoid Hemorrhage with Residual Arachnoid Cyst: A Case Report
}

\author{
Eda DERLE', Ilkin IYIGUNDOGDU1', Cem YILMAZ² \\ ${ }^{1}$ Baskent University, School of Medicine, Department of Neurology, Ankara, Turkey \\ ${ }^{2}$ Baskent University, School of Medicine, Department of Neurosurgery, Ankara, Turkey
}

\section{ABSTRACT}

Spinal subarachnoid hemorrhage is a rare condition that usually occurs because of secondary causes and idiopathic spontaneous ones are extremely rare. We report a 56 -year-old woman who presented with severe headache, neck pain and urinary retention. Magnetic resonance imaging revealed subarachnoid hemorrhage that extended from T1 to lumbar vertebrae. She was treated conservatively and symptoms were resolved within 1 month. An asymptomatic arachnoid cyst was observed on the follow-up magnetic resonance imaging after 3 months with no neurologic deterioration. She was still stable clinically and radiologically at the end of 19 months. Spinal subarachnoid hemorrhage is a surgical emergency, although in some cases a conservative approach may be useful.

KEYWORDS: Spinal subarachnoid hemorrhage, Arachnoid cyst, Spontaneous

\section{INTRODUCTION}

$O$ pinal subarachnoid hemorrhage (SSH) is a rare condition that comprise $0.05-1.5 \%$ of all cases of subarachnoid hemorrhage in the literature (7). SSH usually occurs because of secondary causes such as trauma, vascular lesions, spinal cord tumors, coagulation abnormalities, lumbar puncture and vasculitis $(3,7)$. It can also occur spontaneously with unknown pathogenesis which is extremely rare.

Spinal arachnoid cyst due to arachnoiditis after subarachnoid hemorrhage $(\mathrm{SAH})$ is also rare and usually presents as deterioration of neurological condition after several weeks to years from initial event (1).

In this report, we presented a rare case of idiopathic spinal subarachnoid hemorrhage in a 56 year-old woman which developed and regressed spontaneously with residual arachnoid cyst.

\section{CASE REPORT}

A 56-year-old female patient with a previous history of hypertension presented to another hospital with abrupt onset severe back pain, headache and urinary retention. Initial brain computerized tomography (CT) was unremarkable and she received medical treatment for pain. Her back pain temporary improved but difficulty in urination continued. She presented to our neurology department 10 days from the onset of the symptoms. At the first evaluation, neurological examination was normal except for neck stiffness and globe vesicale. Brain CT showed no abnormality. For further evaluation, magnetic resonance imaging (MRI) of brain and spinal cord revealed diffuse high signal intensity mass, both ventral and dorsal to the spinal cord, on T1-weighted images from $\mathrm{T} 1$ to L1 that was compatible with subacute subarachnoid hemorrhage (Figure 1A,B). Spinal angiography was performed and showed no vascular malformation. Also no coagulation disorder or systemic vasculitis was detected with extensive evaluation. We decided to follow up the patient conservatively and pregabalin was started for her back pain. The patient's 
symptoms recovered after conservative treatment except bladder dysfunction and she was discharged with urinary catheter after 7 days. She had no bladder or bowel dysfunction and her pain had relieved when she admitted 1 month later and follow-up MRI showed sequela hemorrhage between
T6 and T9 especially anterior of the spinal cord (Figure 2A). Follow-up MRI at the third month revealed cystic cavitation in the anterior subarachnoid space of T3-4, T5-6 and T8-9 and she had no additional neurological symptom (Figure 2B). She did not complain about any clinical symptoms for 8

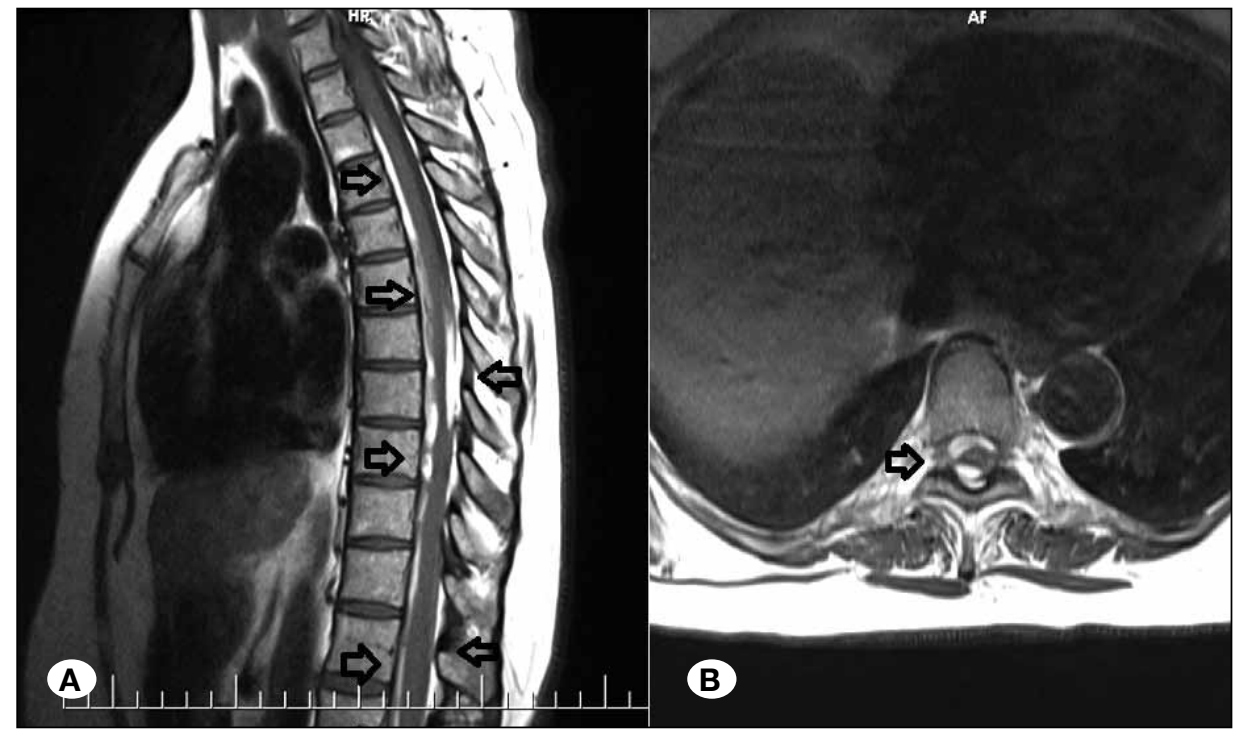

Figure 1: A) T1 weighted sagittal sequences, B) T1 weighted axial sequences. Thoracic MRI revealed diffuse high signal intensity mass extended from $\mathrm{T} 1$ to lumbar region that was compatible with subacute subarachnoid hemorrhage.
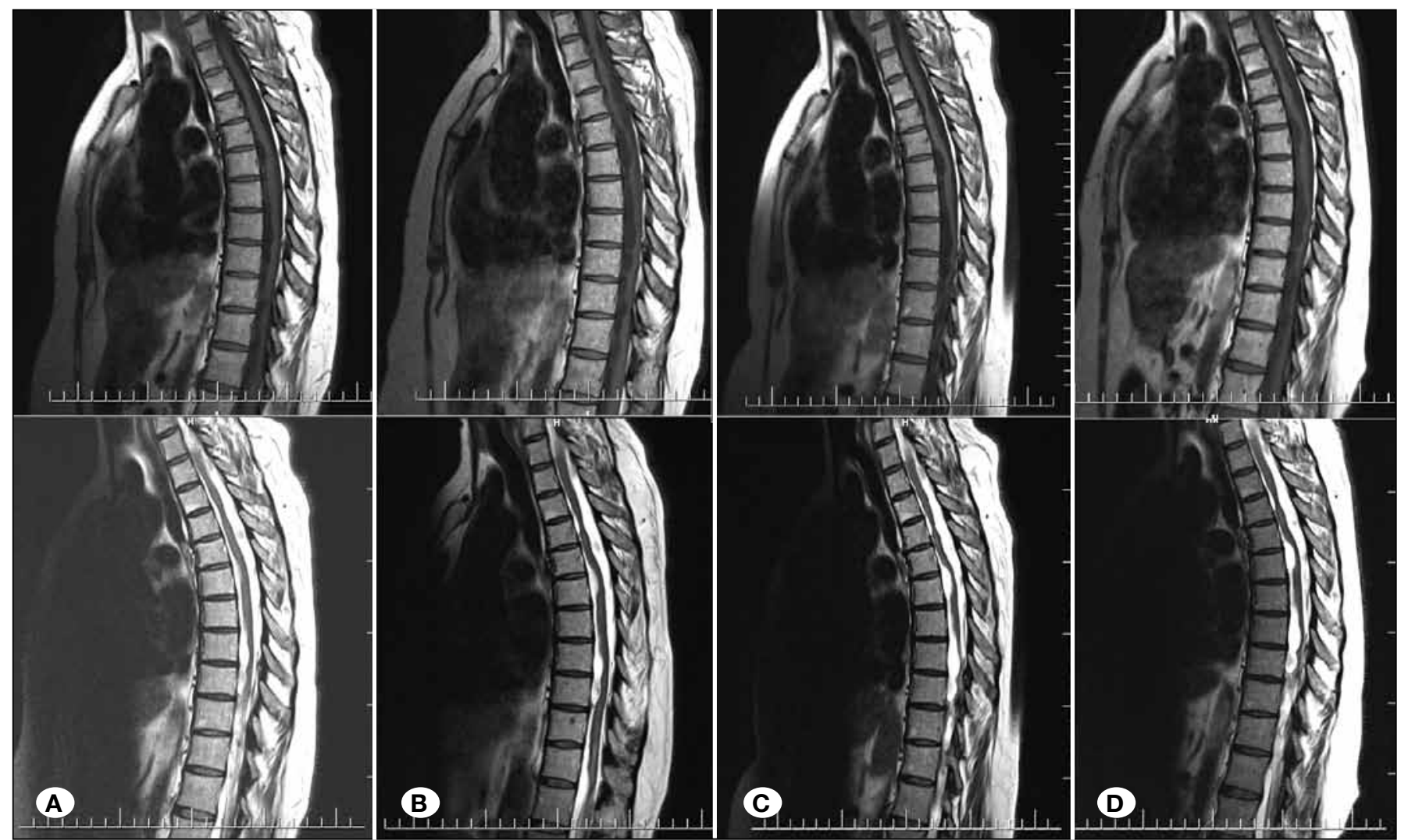

Figure 2: Thoracic MRI (T1 and T2 weighted sagittal sequences). A) Sequel hemorrhage between T6-T9 especially anterior to the cord. B) Cystic cavitation in the anterior subarachnoid space of T3-4, T5-6 and T8-9 at the third month. C) Cystic expansion in the anterior subarachnoid area at the level of T3-4, T5-6 and T8-9 and indentations to the cord at the 8 month and D) no further changes at the 19 month. 
months after initial diagnosis but MRI findings showed cystic expansion in the anterior subarachnoid area at the level of T34, T5-6 and T8-9 and indentations to the spinal cord (Figure $2 \mathrm{C})$. The view of the spinal cord was irregular and atrophic and after 19 months from initial examination. MRI and neurological examination were stable (Figure 2D).

\section{DISCUSSION}

Spontaneous SSH is an extremely rare condition and to the best of our knowledge, there are less than 25 cases, including ours in the literature $(3,10)$. Secondary cases caused by trauma, iatrogenic (lumbar puncture), vascular malformations, spinal tumors, coagulation or hematological pathologies are more frequent than spontaneous SSH (3). Our patient was diagnosed with spinal spontaneous hemorrhage since no radiological finding suggest vascular pathology or tumor and she had no evidence of coagulopathy. Moreover, she had no antecedent iatrogenic procedure. Domenicucci et al. collected 69 SSH cases between 1960-2003 and 12 of 69 (17.3\%) cases were classified as spontaneous because no correlation was found with any of the causing factor (3). The underlying mechanisms or the origin of the bleeding responsible for the $\mathrm{SSH}$ have still not been established and presumed as the causes of bleeding are rupture of the arteries and radicular veins $(3,7)$. Another suggested mechanism was the rupture of the valveless radiculomedullary veins that cross the subdural and subarachnoid space in case of a sudden increase of intraabdominal or intrathoracic pressure such as coughing $(7,10)$.

SSH presents as severe back pain, headache, paraparesis, sensory or sphincter disturbance depends on the level of the lesion $(2,10,13)$. Cerebral symptoms after SSH are common because of the migration of blood and it has been suggested that approximately $80 \%$ of patients have concomitant intracranial symptoms such as headache $(70 \%)$ and mental changes (22\%) (13). Moreover, especially lesions involving the upper cervical spine may present as intracranial subarachnoid hemorrhage $(5,14)$.

MRI plays an important role for diagnosis and follow-up the extension of the hemorrhage and to detect the relationship between the hemorrhage and the spinal cord (9). However extra-arachnoid and subarachnoid spaces are so close that sometimes the diagnosis cannot be confirmed (12). CT is also useful, but not usually diagnostic in SSH $(3,12)$. Also spinal angiography is necessary to rule out the underlying vascular disease (9). But in cases that emergency decompressive surgery is necessary, spinal MRI may also give information about probable vascular malformation $(6,9)$.

SSH may cause spinal cord and nerve root compression and usually is considered as a surgical emergency $(3,6)$. Surgical decompression is the main treatment in SSHs, but there are also few case reports that were conservatively treated $(3,6,9,10)$. Komiyama et al. suggested that ventral type usually has a benign course and shows no neurological deficits and surgical decompression may be unnecessary for ventral SSHs (9). However the location of the hemorrhage is not the only factor in determining the treatment approaches. The decision for surgery depends generally on the neurological deficit of the patient. In previous reports although the hemorrhage was located rather than ventral subarachnoid space, patients recovered without surgical decompression $(6,11)$. Similar to those cases, our patient has both ventral and dorsal hemorrhage in the thoracic and lumbar spinal cord from T1 to L1, followed up without surgery and recovered by conservative management.

In follow-up period, in consecutive MRI of the spinal column, we also observed cystic changes without any deterioration in neurological examination. Arachnoid cyst development after SAH is also rare, and most of the reported cases are after cerebral SAH (1). Arachnoid cysts can be asymptomatic or can cause neurological deterioration $(4,12)$. Surgical decompression and shunt placement are the most common procedures in the treatment (1). But conservative management is also recommended in some cases $(4,8)$.

\section{CONCLUSION}

Spinal subarachnoid hemorrhage is a rare lesion which is difficult to diagnose. Although there has been spontaneous recovery in several cases with conservative treatment or close follow-up, it is potentially dangerous and may have disastrous consequences, so SSH should always be considered in case of sudden back pain associated with severe headache.

\section{- REFERENCES}

1. Basaran R, Kaksi M, Efendioglu M, Onoz M, Balkuv E, Kaner T: Spinal arachnoid cyst associated with arachnoiditis following subarachnoid haemorrhage in adult patients: A case report and literature review. Br J Neurosurg 29(2):285-289,2015

2. Bruce-Brand RA, Colleran GC, Broderick JM, Lui DF, Smith EM, Kavanagh EC, Poynton AR: Acute non-traumatic spinal intradural hematoma in a patient on warfarin. $\mathrm{J}$ Emerg Med 45: 695-697, 2013

3. Domenicucci M, Ramieri A, Paolini S, Russo N, Occhiogrosso G, Di Biasi C, Delfini R: Spinal subarachnoid hematomas: Our experience and literature review. Acta Neurochir (Wien) 147: 741-750; discussion 750, 2005

4. Ginanneschi F, Palma L, Rossi A: Arachnoid cyst and arachnoiditis following idiopathic spinal subarachnoid haemorrhage. Br J Neurosurg 22: 578-579, 2008

5. Gonzalez LF, Zabramski JM, Tabrizi P, Wallace RC, Massand MG, Spetzler RF: Spontaneous spinal subarachnoid hemorrhage secondary to spinal aneurysms: Diagnosis and treatment paradigm. Neurosurgery 57:1127-1131; discussion 1127-1131, 2005

6. Kim JS, Lee SH: Spontaneous spinal subarachnoid hemorrhage with spontaneous resolution. J Korean Neurosurg Soc 45: 253-255, 2009

7. Kim YH, Cho KT, Chung CK, Kim HJ: Idiopathic spontaneous spinal subarachnoid hemorrhage. Spinal Cord 42:545-547, 2004

8. Kok AJ, Verhagen WI, Bartels RH, van Dijk R, Prick MJ: Spinal arachnoiditis following subarachnoid haemorrhage: report of two cases and review of the literature. Acta Neurochir (Wien) 142: 795-798; discussion 798-799, 2000 
9. Komiyama M, Yasui T, Sumimoto T, Fu Y: Spontaneous spinal subarachnoid hematoma of unknown pathogenesis: Case reports. Neurosurgery 41: 691-693; discussion 693-694, 1997

10. Oji Y, Noda K, Tokugawa J, Yamashiro K, Hattori N, Okuma Y: Spontaneous spinal subarachnoid hemorrhage after severe coughing: A case report. J Med Case Rep 7:274, 2013

11. Ruelle A, Zerbi D, Andrioli G: Spinal subarachnoid bleeding of unknown etiology. Case reports. J Neurosurg Sci 45:53-57, 2001
12. Sasaji T, Shinagawa K, Matsuya S: Spontaneous thoracic spinal subarachnoid hemorrhage diagnosed with brain computed tomography. Tohoku J Exp Med 231:139-144, 2013

13. Sunada I, Akano Y, Kidosaki Y, Shimokawa N, Yamamoto S: Spontaneous spinal subarachnoid hematoma-case report. Surg Neurol 44:133-136, 1995

14. Yaldizli O, Euler S, Willi B, Wiesbeck GA, Wurst FM: Spontaneous spinal subarachnoid haemorrhage-a complication of alcohol withdrawal therapy. Drug Alcohol Rev 27:429-432, 2008 